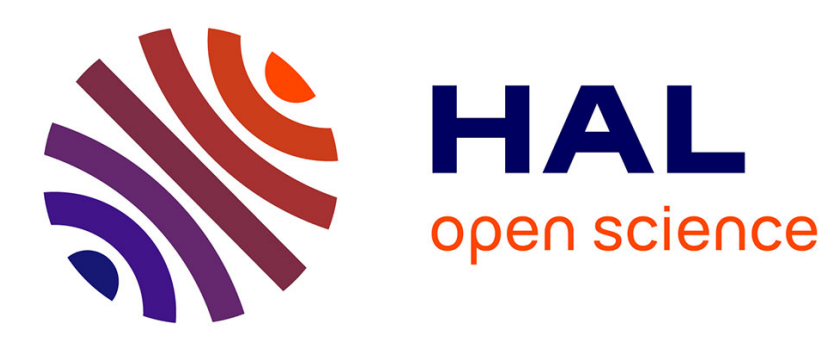

\title{
Dispersal, environmental forcing, and parasites combine to affect metapopulation synchrony and stability
}

\author{
Alison Duncan, Andrew Gonzalez, Oliver Kaltz
}

\section{To cite this version:}

Alison Duncan, Andrew Gonzalez, Oliver Kaltz. Dispersal, environmental forcing, and parasites combine to affect metapopulation synchrony and stability. Ecology, 2015, 96, pp.284-290. 10.1890/140137.1. hal-02353403

\section{HAL Id: hal-02353403 \\ https://hal.umontpellier.fr/hal-02353403}

Submitted on 7 Nov 2019

HAL is a multi-disciplinary open access archive for the deposit and dissemination of scientific research documents, whether they are published or not. The documents may come from teaching and research institutions in France or abroad, or from public or private research centers.
L'archive ouverte pluridisciplinaire HAL, est destinée au dépôt et à la diffusion de documents scientifiques de niveau recherche, publiés ou non, émanant des établissements d'enseignement et de recherche français ou étrangers, des laboratoires publics ou privés. 


\title{
Dispersal, environmental forcing, and parasites combine to affect metapopulation synchrony and stability
}

\author{
Alison B. Duncan, ${ }^{1,3}$ Andrew Gonzalez, ${ }^{2}$ and Oliver Kaltz ${ }^{1}$ \\ ${ }^{1}$ Institut des Sciences de l'Evolution, UMR 5554, Université Montpellier 2, Place Eugene Bataillon, \\ 34095 Montpellier Cedex 05, France \\ ${ }^{2}$ Department of Biology, McGill University, 1205 Avenue Docteur Penfield, Montreal, Quebec H3A 1 B1 Canada
}

\begin{abstract}
Dispersal can have positive and negative effects on metapopulation stability and persistence. One prediction is that high levels of dispersal synchronize density fluctuations between subpopulations. However, little is still known about how biotic and abiotic factors combine to modify the effects of dispersal rate on synchrony and metapopulation dynamics.

In a fully factorial experimental design, we investigated the combined effects of (1) dispersal, (2) parasite infection, and (3) synchrony in temperature fluctuations on subpopulation synchrony, metapopulation instability, and minimum population size, in laboratory metapopulations of the ciliate Paramecium caudatum.

Metapopulations, comprising two subpopulations linked by high or low levels of dispersal, were exposed to daily fluctuations in temperature between permissive $\left(23^{\circ} \mathrm{C}\right)$ and restrictive $\left(5^{\circ} \mathrm{C}\right)$ conditions. Infected metapopulations started the experiment with one subpopulation uninfected, while the other was infected at a prevalence of $5 \%$ with the bacterial parasite Holospora undulata. The temperature synchrony treatment involved subpopulations within a metapopulation following the same (synchronous temperatures) or different (asynchronous temperatures) temporal sequences. Population size was tracked over the 56-day experiment.

We found that subpopulation density fluctuations were synchronized by high dispersal in infected metapopulations, and by synchronous temperatures in all metapopulations. Subpopulation synchrony was positively correlated with metapopulation instability and minimum metapopulation size, highlighting the multiple consequences of our treatments for metapopulation dynamics.

Our results illustrate how parasites can generate dispersal-driven synchrony in non-cycling, declining populations. This "biotic forcing" via a natural enemy added to the temperaturedependent environmental forcing. We therefore conclude that predictions of metapopulation persistence in natural populations require simultaneous investigation of multiple ecological and epidemiological factors.
\end{abstract}

Key words: dispersal; epidemiology; extinction; Holospora undulata; metapopulation; microcosm; Paramecium caudatum; spatiotemporal variation; synchrony.

\section{INTRODUCTION}

Metapopulation persistence is the result of the interplay between dispersal and stochastic processes in the biotic and abiotic environment. Generally, dispersal can rescue spatially isolated populations at low density and therefore be essential for long-term metapopulation persistence (Hanski 1991, Gonzalez et al. 1998). Yet it is a double-edged sword (Hudson and Cattadori 1999), as too much dispersal can increase population synchrony (Bjornstad et al. 1999, Gouhier et al. 2010), thereby increasing the risk of metapopulation-wide extinction when densities are low. This synchronizing effect of high dispersal has been demonstrated in laboratory (Dey and Joshi 2006, Vogwill et al. 2009) and field (Matter and

Manuscript received 22 January 2014; revised 29 May 2014; accepted 25 June 2014. Corresponding Editor: A. M. Kilpatrick.

${ }^{3}$ E-mail: Alison.duncan@univ-montp2.fr
Roland 2010) populations. Thus, low to intermediate levels of dispersal are expected to lead to longer persistence times, all else being equal.

However, dispersal will rarely act in isolation. Natural enemies (parasites, predators) are also potential drivers of population synchrony that may destabilize metapopulations through "biotic forcing". This occurs when dispersal spreads parasites or predators across a metapopulation (Ostfeld et al. 2005), thereby generating parallel mortality levels across the landscape (Ims and Andreassen 2000, Fontaine and Gonzalez 2005, Gouhier et al. 2010). Thus, the presence of natural enemies may strengthen the synchronizing effect of dispersal (Vasseur and Fox 2009, Vogwill et al. 2009).

Moreover, the effects of dispersal may also interact with abiotic conditions. It is established that synchronous environmental fluctuations can force populations into synchrony (Hudson and Cattadori 1999, Matthews and Gonzalez 2007, Abbott 2011). This "Moran effect" 
(Royama 1992) can counterbalance the stabilizing effects of dispersal (Abbott 2011), because dispersal between synchronous populations cannot induce a rescue effect where migration might save a subpopulation from extinction. Therefore, synchronous abiotic fluctuations, in addition to effects of dispersal, can lead to even stronger population synchrony and greatly reduced metapopulation persistence (Heino et al. 1997).

Integrating the relative contributions from spatial connectivity by dispersal, species interactions, and environmental stochasticity for population stability and persistence into a general metacommunity framework remains a major challenge (Ruokolainen 2013). However, laboratory microcosms represent a powerful experimental approach to study these effects alone and in combination (Jessup et al. 2004). For example, a recent study found that dispersal synchronized population cycles of the protozoan Tetrahymena only in the presence of a predator; synchronizing effects of correlated environmental fluctuations acted independently (Vasseur and Fox 2009). In the present study, we used a similar experimental setup, but instead compared how different levels of dispersal, and a parasite instead of a predator, drive population synchrony. Our study therefore represents the first to investigate how different levels of dispersal act to spread a parasite through a metapopulation, from an infected to an uninfected subpopulation, in the presence or absence of synchronous abiotic environmental fluctuations.

The aim of our study was to investigate how parasitism interacted with temperature fluctuations to modify the effects of dispersal on population synchrony and stability. We used simple experimental metapopulations of the ciliate Paramecium caudatum and its bacterial parasite Holospora undulata (Duncan et al. 2013). In a fully factorial design, infected and uninfected metapopulations were exposed to high or low levels of dispersal and synchronous or asynchronous daily fluctuations between restrictive $\left(5^{\circ} \mathrm{C}\right)$ and permissive $\left(23^{\circ} \mathrm{C}\right)$ temperatures. This factorial design permits the quantification of the relative combined and independent contributions of each factor (dispersal, parasite, temperature) on population dynamics. In a previous study, we demonstrated that asynchrony in positively autocorrelated environmental fluctuations ("red noise") enabled infected metapopulations to maintain larger sizes (Duncan et al. 2013). Here, we focus on the synchronizing effect of dispersal and its interaction with biotic and abiotic factors.

We show, for the first time, dispersal-driven synchrony in declining populations under high dispersal in infected metapopulations, and under synchronous temperature fluctuations in all metapopulations. Thus, the highest levels of population instability were present in highly connected, parasite-infected metapopulations, subjected to synchronized environmental fluctuations.

\section{Methods \\ Study organisms}

Paramecium caudatum is found in still freshwater and feeds on bacteria and detritus (Wichterman 1986). We maintained Paramecium in an organic lettuce-based medium, supplemented with Serratia marcescens bacteria as food (Nidelet and Kaltz 2007). Reproduction is predominantly asexual (mitotic division). Optimal growth temperature is around $28^{\circ} \mathrm{C}$, with tolerance ranging from $\sim 2^{\circ} \mathrm{C}$ to $\sim 36^{\circ} \mathrm{C}$ (Krenek et al. 2012).

Holospora undulata, a gram-negative $\alpha$-proteobacterium, infects the micronucleus of $P$. caudatum. Horizontal infection occurs when infectious forms, released into the environment following host division or death, are ingested (Fokin 2004). Vertical transmission occurs by parasite transfer to the micronuclei of mitotic daughter cells. Infection reduces host division and longevity (Nidelet et al. 2009). Parasite development is optimal between $23^{\circ} \mathrm{C}$ and $30^{\circ} \mathrm{C}$ and arrested at $10^{\circ} \mathrm{C}$ (Fels and Kaltz 2006).

\section{Host and parasite material}

We established replicate subpopulations of $30 \mathrm{~mL}$ in 50-mL Falcon tubes, at a density of 250 Paramecium/ $\mathrm{mL}$, from a mass Paramecium culture (clone VEN; Duncan et al. 2010). Infected subpopulations were established by adding infected clone VEN individuals to obtain 5\% initial infection prevalence (see Duncan et al. [2013] for details).

\section{Experimental design}

We created infected and uninfected Paramecium metapopulations, consisting of two subpopulations linked by dispersal; this represents the simplest possible setup, but nonetheless captures the essential ingredients of metapopulation theory (Hanski and Gaggiotti 2004). Infected metapopulations were initiated with one infected and one uninfected subpopulation. Metapopulations were assigned to two treatments. First, the dispersal treatment consisted of linking subpopulations by high $(6.67 \%)$ or low $(0.667 \%)$ dispersal. Second, the temperature synchrony treatment entailed subpopulations experiencing the same (synchronous) or different (asynchronous) temperature fluctuations. The experimental design was fully factorial, with a total of 64 (2 parasite $\times 2$ dispersal $\times 2$ temperature synchrony $\times 8$ replicates) metapopulations.

Dispersal.-Dispersal entailed the transfer of either 2 $\mathrm{mL}(6.67 \%)$ or $200 \mu \mathrm{L}(0.667 \%)$ of culture between subpopulations every 4 days. Levels of dispersal chosen span a range where differences in effects on density synchrony are observed (Fox et al. 2013). Prior to dispersal, $20 \%(6 \mathrm{~mL})$ of the population was culled and subsequently replaced with fresh medium (following dispersal). After day 28, culling increased to every 2 days to enhance population decline (see Duncan et al. 2013). 
Temperature synchrony.-All subpopulations were subjected to temperature fluctuations between $5^{\circ} \mathrm{C}$ and $23^{\circ} \mathrm{C}$, following synchronous or asynchronous (Duncan et al. 2013) random sequences for 56 days (28 days at $23^{\circ} \mathrm{C}$ and $5^{\circ} \mathrm{C}$, respectively). Temperature synchrony was achieved by allocating each subpopulation the same (synchronous temperatures) or different (asynchronous temperatures) temporal sequences. Under synchronous temperatures, the sequences were perfectly correlated between subpopulations $(r=1)$. Asynchronous temperatures entailed subpopulations frequently experiencing contrasting conditions as subpopulations were assigned different sequences (mean temperature correlation coefficient between subpopulation sequences $=-0.03 \pm 0.02$ [mean $\pm \mathrm{SE}], n=32$ subpopulations, $P=0.13$ ).

In a supplementary treatment, we set up eight infected and eight uninfected subpopulations that were not coupled by dispersal. Each population was assigned a different temperature fluctuation sequence. The main purpose of these controls was to compare temporally uncorrelated vs. autocorrelated temperature fluctuations, in the absence of dispersal (Duncan et al. 2013). The degree of synchrony between pairs of sequences was not explicitly controlled and the temperature correlation coefficient ranged from $r=-0.7$ to $r=0.43$.

In both experiments, we measured subpopulation size every 4 days over the 56-day duration of the experiment by counting the number of Paramecium in $150-\mu \mathrm{L}$ samples under a dissecting microscope. The spread of infection was followed in 8-day intervals, as described in Duncan et al. (2013).

\section{Statistical analysis}

Metapopulations with dispersal.-We measured three Paramecium population parameters: (1) Synchrony in population size fluctuations between subpopulations in each metapopulation, calculated as the intraclass correlation coefficient of size, $r_{i}$ ( $\left.\operatorname{Zar} 1999\right)$. If $r_{i}$ is close to 1 , subpopulations are synchronous; if $r_{i}$ is close to 0 , subpopulations are asynchronous (Zar 1999). (2) Variance in mean subpopulation size through time, as a proxy for metapopulation instability. (3) Minimum mean subpopulation size was taken as a proxy for, and is associated with, elevated metapopulation extinction risk. Our lowest population sizes observed were $\sim 1000$ and the highest were $\sim 3000$. In a previous experiment, these population sizes were associated with a $34 \%( \pm 95 \%$ CI $0.263,0.429)$ and $4.6 \%$ ( $\pm 95 \%$ CI $0.010,0.827$ ) risk of extinction, respectively (Duncan et al. 2011).

We used factorial general linear models (GLM) to investigate how dispersal, temperature synchrony, and parasite infection influenced variation in these three variables. The intraclass correlation coefficient was transformed using the Fisher $z$ transformation (Matthews and Gonzalez 2007) and the subpopulation variance was square-root transformed to meet model assumptions and improve model fit. The variance was not significantly correlated with the mean $(r=0.13, n=$ 64, $P=0.3039$ ), and therefore there was no need to normalize the variance by calculating the coefficient of variation. Subpopulations within a metapopulation experienced either uncorrelated temporal fluctuations, or fluctuations that were positively autocorrelated (Duncan et al. 2013). Accordingly, temporal noise structure was included in all models as a main effect.

We used path analysis (Webley and Lea 1997) to explore the direct and indirect links between metapopulation synchrony and variance with minimum metapopulation size. Path coefficients were calculated using standardized beta regression coefficients, which assume variables are normally distributed, which was the case for all variables measured.

Metapopulations without dispersal.-A GLM tested how temperature synchrony and parasite infection influenced metapopulation synchrony, in the absence of dispersal. To this end, hypothetical metapopulations were assembled by considering all possible pairs of infected and uninfected subpopulations. The correlation coefficient for temperature fluctuations between subpopulations was calculated for each pair (as in Benton et al. 2001). This quantitative estimate of temperature synchrony was fitted to the model as a covariate.

All analyses were done using JMP 10 (SAS Institute 2012).

\section{REsults}

\section{Metapopulation size synchrony}

Dispersal, parasite infection, and temperature synchrony all influenced synchrony in size fluctuations between subpopulations. Dispersal and infection had a nonadditive effect on synchrony (significant infection $\times$ dispersal interaction; Table 1). High dispersal increased subpopulation size synchrony in infected populations, but not in uninfected populations (Fig. 1a; see post-hoc in contrasts Table 1). This interaction explained $19 \%$ of the variation in the intraclass correlation coefficient, which was our measure of synchrony.

Synchronized temperature fluctuations also had a strong synchronizing effect on subpopulation sizes (significant effect of temperature synchrony; Table 1, Fig. 1a). The temperature synchrony treatment explained $49 \%$ of the observed variation in subpopulation size synchrony, and there were no significant interactions with dispersal or parasite infection $(P>0.15)$.

The independent effect of temperature synchrony, in the absence of dispersal, was confirmed by the supplementary experiment. Synchronous temperature fluctuations alone enhanced subpopulation size synchrony, as shown by a positive relationship between the correlation coefficient for temperature synchrony and the intraclass correlation coefficient for size synchrony (Table 1, Fig. 2). 
TABLE 1. Effect of dispersal, temperature synchrony, and parasite infection on synchrony between subpopulation density fluctuations, the variance in metapopulation densities, and minimum metapopulation density.

\begin{tabular}{|c|c|c|c|c|c|c|}
\hline & \multicolumn{2}{|c|}{$\begin{array}{c}\text { Intraclass } \\
\text { correlation coefficient }\end{array}$} & \multicolumn{2}{|c|}{ Metapopulation variance } & \multicolumn{2}{|c|}{ Minimum metapopulation size } \\
\hline & df & $F$ ratio & df & $F$ ratio & df & $F$ ratio \\
\hline Parasite infection & 1,57 & 0.22 & 1,58 & 0.03 & 1,59 & $28.49 * * *$ \\
\hline Dispersal & 1,57 & 1.77 & 1,58 & 0.57 & 1,59 & 0.02 \\
\hline Temperature synchrony & 1,57 & $30.06 * * *$ & 1,58 & $9.20 * *$ & 1,59 & $10.45 * *$ \\
\hline Parasite $\times$ dispersal & 1,57 & $11.43 * *$ & 1,58 & $6.91^{*}$ & 1,59 & $3.88 *$ \\
\hline High dispersal parasite vs. rest & 1,57 & $8.96^{* *}$ & 1,58 & 3.44 & 1,59 & $18.51 * * *$ \\
\hline Parasite $\times$ temperature synchrony & 1,57 & 3.65 & & & & \\
\hline Noise color & 1,57 & $5.22 *$ & 1,58 & $7.89 * *$ & & \\
\hline
\end{tabular}

Notes: Synchrony in subpopulation density fluctuations was measured using the intraclass correlation coefficient. A main effect for temporal noise color was included in all maximal models. Statistics shown represent values taken from minimal models only. All terms in models were tested against the error term. Post-hoc contrasts for the parasite $\times$ dispersal interaction term are shown, for each model, where the high-dispersal, parasite-infected metapopulations were compared with all the other metapopulations. Empty cells denote a term not included in the minimal model.

$* P<0.05 ; * * P<0.001 ; * * * P<0.0001$.

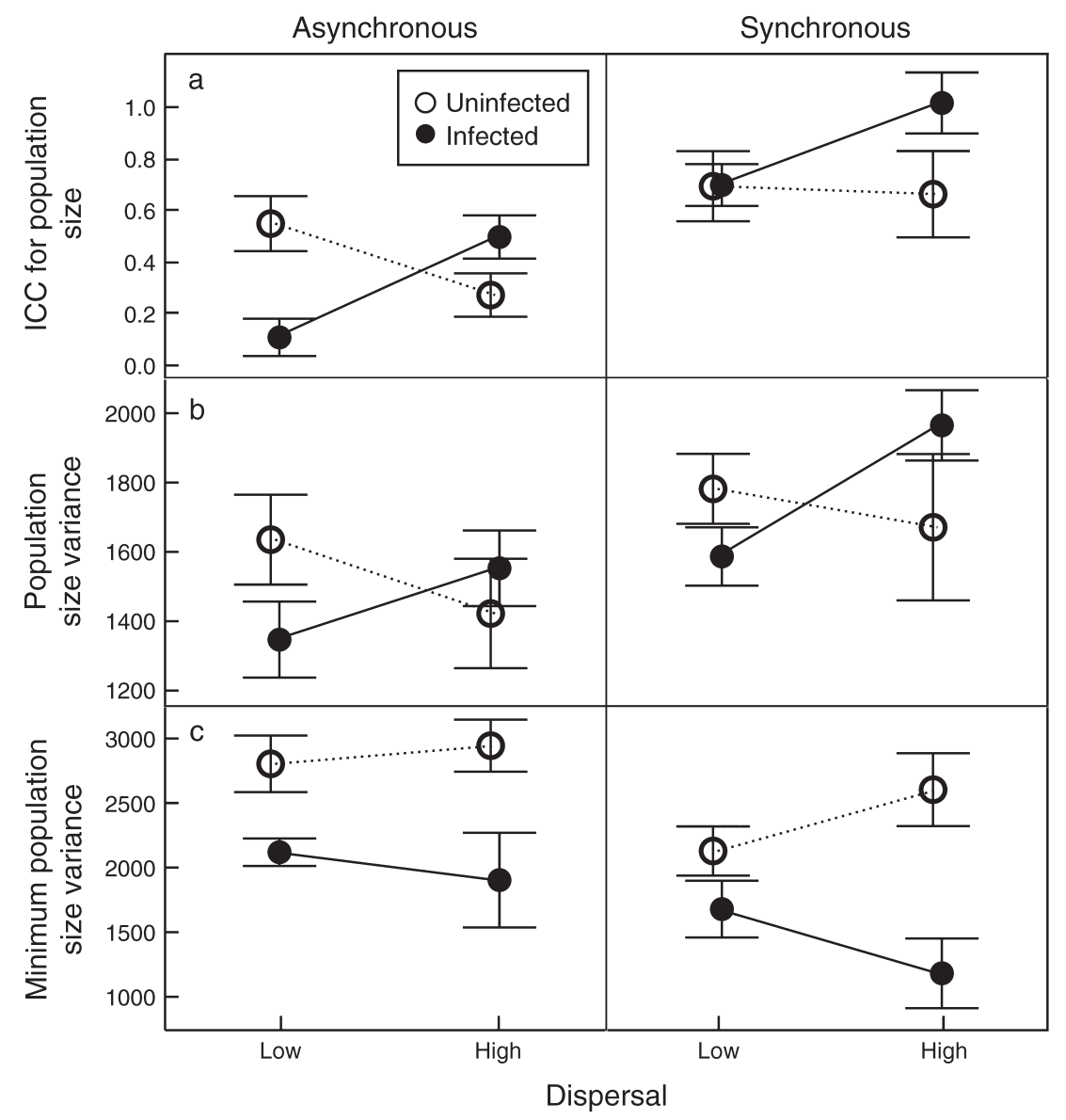

FIG. 1. (a) Synchrony in subpopulation size fluctuations, (b) variance in metapopulation size fluctuations, and (c) minimum metapopulation size in infected (solid circles) and uninfected (open circles) metapopulations experiencing high and low levels of dispersal and synchronous and asynchronous temperature fluctuations. Values are means \pm SE. Synchrony in subpopulation size fluctuations is measured as the intraclass correlation coefficient (ICC; values close to 1 indicate synchronous fluctuations, and values close to 0 uncorrelated fluctuations). Values for (a) were transformed using Fisher's $z$ transformation and values for (b) with the square-root transformation. 


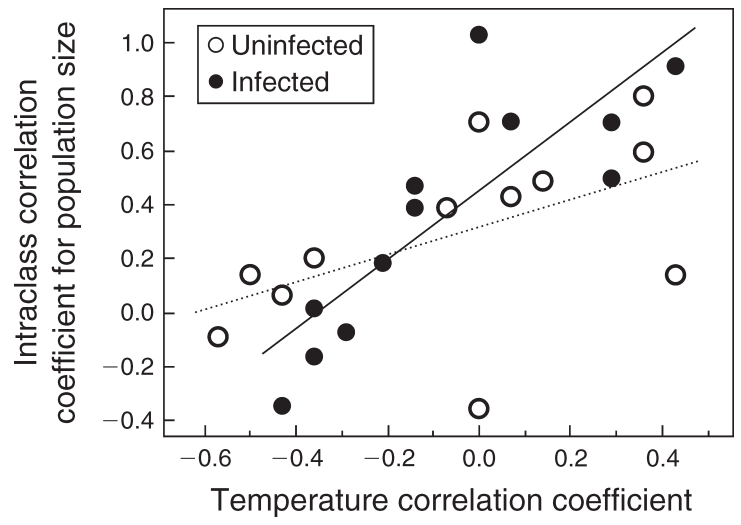

FIG. 2. Relationship between the temperature correlation coefficient and the intraclass correlation coefficient (transformed using Fisher's $z$ transformation) for subpopulation size in infected (open circles) and uninfected (closed circles) metapopulations without dispersal. Synchrony in subpopulation size fluctuations is measured as the intraclass correlation coefficient (note values close to 1 indicate dynamics are synchronous, and close to 0 are uncorrelated).

\section{Variance and minimum metapopulation size}

Both the variance in population size (Fig. 1b) and the minimum population size (Fig. 1c) were markedly affected by the same treatment combinations driving subpopulation synchrony (Table 1). High dispersal compounded the effect of parasite infection, increasing the variance in metapopulation size and reducing the minimum metapopulation size (see post-hoc contrasts in Table 1). Similarly, synchronous temperatures increased population variance and lowered minimum population size.

\section{Relationships between synchrony, metapopulation instability, and minimum size}

Path analysis revealed that both greater levels of subpopulation size synchrony and metapopulation variance were associated with lower minimum metapopulation size (Fig. 3). Indeed, there were direct negative effects of synchrony in subpopulation size fluctuations, and to a lesser degree, metapopulation variance, on minimum metapopulation size. There was also a significant positive correlation between subpopulation size synchrony and variance. These general patterns also held when analyzed separately for dispersal, parasite infection, and temperature synchrony treatments (Appendix).

\section{DisCUSSION}

We found that dispersal, parasite infection, and synchrony in temperature fluctuations all affected metapopulation stability. High levels of dispersal and parasite infection interacted to drive population synchrony and increase variability in metapopulation fluctuations. In contrast, the destabilizing effects of temperature synchrony acted independently of dispersal and parasite infection.

\section{Combined effects of dispersal and parasite infection}

Theory suggests that high levels of dispersal can synchronize subpopulation density fluctuations, which increases metapopulation-wide extinction risk when densities are low (Abbott 2011). Consistent with this idea, our experiment demonstrates that higher dispersal increased synchrony in infected metapopulations. Furthermore, we show that increased population synchrony is linked positively with metapopulation instability and that both are associated with reductions in metapopulation size, and therefore possibly an increased risk of extinction (Fig. 3).

The synchronizing effect of high dispersal was only evident in the presence of parasite infection. This negative combined effect of dispersal and natural enemies was also reported in experiments with bacteria and protists (Vasseur and Fox 2009, Vogwill et al. 2009, Fox et al. 2011, but see Cooper et al. 2012). In our experiment, high levels of dispersal accelerated parasite spread across the metapopulation by almost an entire week, so that prevalence was more similar between subpopulations earlier (see Table 1 and Fig. S1 in Duncan et al. [2013]).

This faster and correlated spread of infection under high dispersal explains why the interconnected subpopulations showed more synchronous changes in subpopulation size. This explanation follows the general idea that high levels of dispersal spread natural enemies more equally between the subpopulations sooner, thereby generating similar mortality across the metapopulation (Fontaine and Gonzalez 2005). This effect of biotic forcing is analogous to environmental forcing (Moran effect), resulting from correlated fluctuations in the abiotic environment. Predator-prey systems often show cyclical population dynamics, which are more conducive to synchronization induced by dispersal (Bjornstad et al.

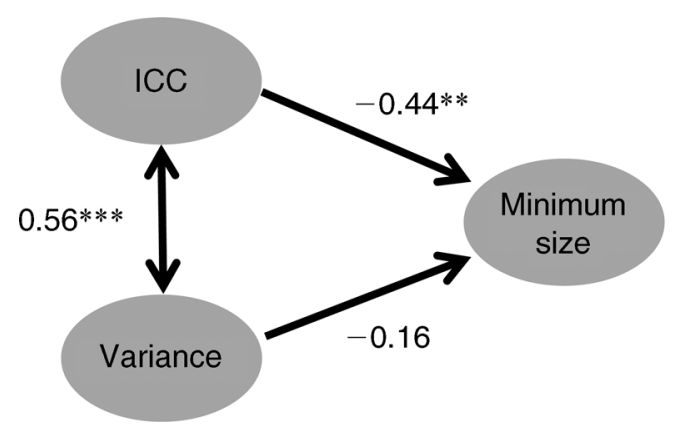

FIG. 3. Direct and indirect effects of the synchrony intraclass correlation coefficient and metapopulation size variance on minimum metapopulation size, as revealed by path analysis. Numbers denote path correlation coefficients calculated using standardized beta regression coefficients across all metapopulations with dispersal.

** $P<0.001 ; * * * P<0.0001$. 
1999, Vasseur and Fox 2009). In our experiment, however, we observed the spread of a single epidemic, which caused a progressive decline in Paramecium population size (Fig. 1; Duncan et al. 2013). This indicates that biotic forcing can also arise without cyclical population dynamics.

\section{Impact of temperature synchrony on Paramecium population size dynamics}

Environmental forcing can produce synchrony in metapopulation dynamics, independently of dispersal (Benton et al. 2001, Vasseur and Fox 2009). As reported previously (Duncan et al. 2013), we found that synchronous temperatures drive population synchrony between subpopulations. Here, we show that this effect acted independently of the rate of dispersal, and occurred even in the absence of dispersal. It was also statistically independent of the effect of parasite infection. Consequently, imposing environmental forcing on highly connected metapopulations under parasite infection resulted in the greatest synchrony and instability.

Furthermore, the impact of temperature synchrony was nearly twice as strong as the combined effect of high dispersal and parasite infection. This may be because fluctuations in environmental temperatures were either uncorrelated $(r=0)$ or totally synchronized $(r=1)$ over the entire metapopulation. Intermediate degrees of synchrony (see Fig. 2) may be necessary to identify interactive effects with dispersal or parasite infection. However, the independent effect of temperature forcing on metapopulation dynamics we observe is consistent with previous findings (Benton et al. 2001, Vasseur and Fox 2009). A recent study showed that the synchronizing effects of dispersal on metapopulations were only affected by environmental forcing over larger spatial scales (Gouhier et al. 2010). Therefore, future experiments should investigate how metapopulations with differing spatial arrangements and dispersal patterns are impacted by the Moran effect.

\section{Implications}

The extrapolation of results from microcosm experiments to real-world scenarios must be done with caution, but they can guide questions for further analysis and modeling. For example, it is becoming increasingly clear that the coupling of species interactions with climatic variables can be important for theoretical models projecting future species distributions (Kubisch et al. 2013). Here, we quantified the relative impact of biotic and abiotic factors and the type of interactions (synergistic vs. additive) between them; this may provide input for the parameterization of such models. Furthermore, our relatively simple experimental scenario with only two subpopulations could be extended to larger metapopulations with more patches, or different spatial arrangements.
Our results also have implications for conservation efforts, often aimed at increasing connectivity between fragmented populations (Crooks and Sanjayan 2006). However, a potential downside of such strategies is that they facilitate the dispersal of natural enemies (Sullivan et al. 2011). Aside from the direct negative effects natural enemies have on population density and extinction risk, they can also act indirectly, by increasing population synchrony, as shown by our experiment. This clearly illustrates the need to incorporate connectivity patterns and dispersal opportunities in models of the spatial dynamics of an epidemic (Viboud et al. 2006, Rohani and King, 2010).

\section{Conclusions}

Microcosm experiments are a powerful tool for testing the fundamental theory of metapopulation dynamics under controlled conditions. Our study confirms the important role of dispersal as a driver of metapopulation synchrony and stability. We also demonstrated that different ecological factors interacted synergistically (parasites) and additively (temperature synchrony) with the effects of dispersal. However, despite this complexity, we validated theory by showing that population synchrony affects metapopulation instability. The robustness of this causal coupling across different treatments underscores the general importance of spatiotemporal dynamics for our understanding of long-term metapopulation persistence.

\section{ACKNOWLEDGMENTS}

We thank Claire Barbera for help in the laboratory, and two anonymous reviewers for very helpful comments on the manuscript. This work was supported by grants from the Agence National de la Recherche ("ANR-09-BLAN-0099", A. Duncan and O. Kaltz). A. Gonzalez is supported by the Canada Research Chair program. This is ISEM contribution no. 2014-082.

\section{Literature Cited}

Abbott, K. C. 2011. A dispersal-induced paradox: synchrony and stability in stochastic metapopulations. Ecology Letters 14(11):1158-1169.

Benton, T. G., C. T. Lapsley, and A. P. Beckerman. 2001. Population synchrony and environmental variation: an experimental demonstration. Ecology Letters 4(3):236-243.

Bjornstad, O. N., R. A. Ims, and X. Lambin. 1999. Spatial population dynamics: analyzing patterns and processes of population synchrony. Trends in Ecology and Evolution 14(11):427-432.

Cooper, J. K., J. Li, and D. J. S. Montagnes. 2012. Intermediate fragmentation per se provides stable predator-prey metapopulation dynamics. Ecology Letters 15(8):856-863.

Crooks, K., and M. A. Sanjayan. 2006. Connectivity conservation. Cambridge University Press, Cambridge, UK.

Dey, S., and A. Joshi. 2006. Stability via asynchrony in Drosophila metapopulations with low migration rates. Science 312(5772):434-436.

Duncan, A. B., S. Fellous, R. Accot, M. Alart, K. Chantung Sobandi, A. Cosiaux, and O. Kaltz. 2010. Parasite-mediated protection against osmotic stress for Paramecium caudatum infected by Holospora undulata is host genotype specific. FEMS Microbiology Ecology 74(2):353-360. 
Duncan, A. B., S. Fellous, and O. Kaltz. 2011. Tempora variation in temperature determines disease spread and maintenance in Paramecium microcosm populations. Proceedings of the Royal Society B 278(1723):3412-3420.

Duncan, A. B., A. Gonzalez, and O. Kaltz. 2013. Stochastic environmental fluctuations drive epidemiology in experimental host-parasite metapopulations. Proceedings of the Royal Society B 280:20131747.

Fels, D., and O. Kaltz. 2006. Temperature-dependent transmission and latency of Holospora undulata, a micronucleusspecific parasite of the ciliate Paramecium caudatum. Proceedings of the Royal Society B 273(1589):1031-1038.

Fokin, S. I. 2004. Bacterial endocytobionts of ciliophora and their interactions with the host cell. International Review of Cytology, Supplement 236:181-249.

Fontaine, C., and A. Gonzalez. 2005. Population synchrony induced by resource fluctuations and dispersal in an aquatic microcosm. Ecology 86(6):1463-1471.

Fox, J. W., G. Legault, D. A. Vasseur, and J. A. Einarson. 2013. Nonlinear effect of dispersal rate on spatial synchrony of predator-prey cycles. PLoS ONE 8(11):e79527.

Fox, J. W., D. A. Vasseur, S. Hausch, and J. Roberts. 2011. Phase locking, the Moran effect and distance decay of synchrony: experimental tests in a model system. Ecology Letters 14(2):163-168.

Gonzalez, A., J. H. Lawton, T. M. Gilbert, T. M. Blackburn, and I. Evanns-Freke. 1998. Metapopulation dynamics, abundance, and distribution in a microecosystem. Science 281(5385):2045-2047.

Gouhier, T. C., F. Guichard, and A. Gonzalez. 2010. Synchrony and stability of food webs in metacommunities. American Naturalist 175(2):E16-E34.

Gouhier, T. C., F. Guichard, and B. A. Menge. 2010. Ecological processes can synchronize marine population dynamics over continental scales. Proceedings of the National Academy of Sciences USA 107(18):8281-8286.

Hanski, I. 1991. Single-species metapopulation dynamics: concepts, models and observations. Biological Journal of the Linnean Society 42(1-2):17-38.

Hanski, I., and O. E. Gaggiotti. 2004. Ecology, genetics and evolution of metapopulations. Elsevier, San Diego, California, USA.

Heino, M., V. Kaitala, E. Ranta, and J. Lindstrom. 1997. Synchronous dynamics and rates of extinction in spatially structured populations. Proceedings of the Royal Society B 264(1381):481-486.

Hudson, P. J., and I. Cattadori. 1999. The Moran effect: a cause of population synchrony. Trends in Ecology and Evolution 5347(98):98-99.

Ims, R. A., and H. P. Andreassen. 2000. Spatial synchronization of vole population dynamics by predatory birds. Nature 408(6809): 194-196.

Jessup, C. M., R. Kassen, S. E. Forde, B. Kerr, A. Buckling, P. B. Rainey, and B. J. M. Bohannan. 2004. Big questions, small worlds: microbial model systems in ecology. Trends in Ecology and Evolution 19(4):189-197.
Krenek, S., T. Petzoldt, and T. U. Berendonk. 2012. Coping with temperature at the warm edge: patterns of thermal adaptation in the microbial eukaryote Paramecium caudatum. PLOS ONE 7(3).

Kubisch, A., R. D. Holt, H. Poethke, and E. A. Fronhofer. 2013. Where am I and why? Synthesizing range biology and the eco-evolutionary dynamics of dispersal. Oikos 122:15321540.

Matter, S. F., and J. Roland. 2010. Local extinction synchronizes population dynamics in spatial networks. Proceedings of the Royal Society B 277(1682):729-737.

Matthews, D. P., and A. Gonzalez. 2007. The inflationary effects of environmental fluctuations ensure the persistence of sink metapopulations. Ecology 88(11):2848-2856.

Nidelet, T., and O. Kaltz. 2007. Direct and correlated responses to selection in a host-parasite system: testing for the emergence of genotype specificity. Evolution 61(8):18031811.

Nidelet, T., J. C. Koella, and O. Kaltz. 2009. Effects of shortened host life span on the evolution of parasite life history and virulence in a microbial host-parasite system. BMC Evolutionary Biology 9:65.

Ostfeld, R. S., G. E. Glass, and F. Keesing. 2005. Spatial epidemiology: an emerging (or re-emerging) discipline. Trends in Ecology and Evolution 20(6):328-336.

Rohani, P., and A. A. King. 2010. Never mind the length, feel the quality: the impact of long-term epidemiological data sets on theory, application and policy. Trends in Ecology and Evolution 25(10):611-618.

Royama, T. 1992. Analytical population dynamics. Chapman and Hall, London, UK.

Ruokolainen, L. 2013. Spatio-temporal environmental correlation and population variability in simple metacommunities. PLoS ONE 8(8):e72325.

SAS Institute. 2012. JMP version 10.1. SAS Institute, Cary, North Carolina, USA.

Sullivan, L. L., B. L. Johnson, L. A. Brudvig, and N. M. Haddad. 2011. Can dispersal mode predict corridor effects on plant parasites? Ecology 92(8):1559-1564.

Vasseur, D. A., and J. W. Fox. 2009. Phase-locking and environmental fluctuations generate synchrony in a predator-prey community. Nature 460(7258):1007-1010.

Viboud, C., O. N. Bjørnstad, D. L. Smith, L. Simonsen, M. A. Miller, and B. T. Grenfell. 2006. Hierarchies in the spread of influenza. Science 312(5543):447-451.

Vogwill, T., A. Fenton, and M. A. Brockhurst. 2009. Dispersal and natural enemies interact to drive spatial synchrony and decrease stability in patchy populations. Ecology Letters 12(11):1194-1200.

Webley, P., and S. Lea. 1997. Advanced statistics: multivariate analysis II: manifest variables analyses. http://people.exeter. ac.uk/SEGLea/multvar2/pathanal.html

Wichterman, R. 1986. The biology of Paramecium. Plenum Press, New York, New York, USA.

Zar, J. H. 1999. Biostatistical analysis. Pearson, Upper Saddle River, New Jersey, USA.

\section{Supplemental Material}

\section{Ecological Archives}

The Appendix is available online: http://dx.doi.org/10.1890/14-0137.1.sm 